\title{
Systematic review of observational studies of the impact of cardiovascular risk factors on preeclampsia in sub-saharan Africa
}

\author{
Oleg Iris Hounkpatin 1,2,3,4, Salimanou Ariyoh Amidou ${ }^{1,2,3,4^{*}}$ (D), Yessito Corine Houehanou ${ }^{1,2,3,4}$, Philippe Lacroix ${ }^{1,2,3,5}$, \\ Pierre Marie Preux ${ }^{1,2,3}$, Dismand Stephan Houinato ${ }^{1,2,3,4}$ and Holy Bezanahary ${ }^{1,2,3,6}$
}

\begin{abstract}
Background: Maternal mortality is a public health issue, particularly in low- and middle-income countries (LMIC). Sub-Saharan Africa (SSA) is the region most affected worldwide by maternal mortality, and preeclampsia is one of the main causes. We performed a systematic review of observational studies to identify the impact of cardiovascular risk factors on preeclampsia in SSA with a more representative sample.

Methods: Databases: PubMed and Google Scholar were searched to identify published studies. Studies were included if they reported results on the link between at least one cardiovascular risk factor and preeclampsia. Relevant studies quality was assessed with the Newcastle-Ottawa Scale (NOS). Odds ratios and relative risk (RR) were reported with their confidence intervals.

Results: Twelve articles (8 case-controls, 3 cohorts, 1 cross-sectional) were included in this review, with a total of 24, 369 pregnant women. Cardiovascular risk factors such as chronic hypertension, overweight, obesity, diabetes and alcohol were significantly associated with a high risk of preeclampsia. Very few data were available concerning some risk factors. None of the articles reported tobacco consumption as a preeclampsia risk factor. There is a lack of data from French-speaking SSA countries.

Conclusion: Cardiovascular risk factors increase the risk of preeclampsia. Our results suggest the need for prospective cohort studies to ascertain this association in order to reduce maternal mortality due to preeclampsia.
\end{abstract}

Keywords: Risk factors, Preeclampsia, Systematic review, Sub-Saharan Africa

\section{Background}

Maternal mortality is a public health issue, particularly in low- and middle-income countries (LMIC). Although the number of maternal deaths worldwide has decreased by $45 \%$ since 1990,800 women still die every day from largely preventable causes before, during and after childbirth, with disparities by region [1]. Sub-Saharan Africa

\footnotetext{
* Correspondence: salmaneamidou@gmail.com

'INSERM, U1094, Tropical Neuroepidemiology, Limoges, France

${ }^{2}$ Univ. Limoges, U1094, Tropical Neuroepidemiology, Institute of Epidemiology and Tropical Neurology, GEIST, Limoges, France

Full list of author information is available at the end of the article
}

(SSA) is the most affected region, with $66 \%$ of maternal deaths. Hypertensive disorders during pregnancy were identified as the second leading cause, after hemorrhage, of maternal and perinatal death, accounting for 14 and 27.1\%, respectively [2]. According to the World Health Organization (WHO), 16\% of maternal deaths in subSaharan Africa are attributable to hypertensive disorders during pregnancy, with preeclampsia and eclampsia being the leading causes [3]. The incidence of preeclampsia ranges from 3 to $5 \%$ [4] and can reach 10\% [5], depending on the region, and remains higher in LMIC. Preeclampsia increases maternal and perinatal mortality

(C) The Author(s). 2021 Open Access This article is licensed under a Creative Commons Attribution 4.0 International License, which permits use, sharing, adaptation, distribution and reproduction in any medium or format, as long as you give appropriate credit to the original author(s) and the source, provide a link to the Creative Commons licence, and indicate if changes were made. The images or other third party material in this article are included in the article's Creative Commons licence, unless indicated otherwise in a credit line to the material. If material is not included in the article's Creative Commons licence and your intended use is not permitted by statutory regulation or exceeds the permitted use, you will need to obtain permission directly from the copyright holder. To view a copy of this licence, visit http://creativecommons.org/licenses/by/4.0/. The Creative Commons Public Domain Dedication waiver (http://creativecommons.org/publicdomain/zero/1.0/) applies to the data made available in this article, unless otherwise stated in a credit line to the data. 
through both fetal and maternal complications. In the literature, authors reported the involvement of many risk factors; the most frequently cited are advanced age, multiple pregnancies, nulliparity, personal history of preeclampsia and pre-pregnancy medical conditions such as chronic high blood pressure, type 2 diabetes and renal failure $[6,7]$. In the last years, with the spread of cardiovascular diseases, many studies have reported that cardiovascular risk factors are involved in the onset of preeclampsia [8-11]. However, most of these studies were conducted in developed countries. In SSA, a large majority were conducted in English-speaking countries. To estimate the real contribution of cardiovascular risk factors, it is necessary to provide representative data. Moreover, especially about SSA countries, that have one of the highest maternal mortality rates worldwide. Since most of these risk factors are preventable, early identification of women at risk appears to be mandatory for optimal management.

This study aimed to systematically review all observational evidence regarding the impact of cardiovascular risk factors on preeclampsia in SSA.

\section{Methods}

\section{Search strategy}

This review was conducted according to PRISMA guidelines [12]. The study protocol was approved by the review team before searching was performed. Studies were retrieved through internet research in PubMed and Google Scholar databases from March 6th to April 30th, 2019. Searching was performed by using terms in line with the "PICO" method. The search was done with the following terms: hypertension, diabetes mellitus, overweight, obesity, tobacco use, alcohol consumption, dyslipidemia, preeclampsia, hypertensive disorders, Africa South of Sahara, and the names of all SSA countries. Keywords were used as follow: (("hypertension" OR "diabetes mellitus" OR "overweight" OR "obesity" OR "alcohol consumption" OR "tobacco use" OR "dyslipidemia") AND (preeclampsia OR "hypertensive disorders")) and all SSA countries names. References of relevant studies were manually screened for additional articles. Titles and abstracts were screened first. Then a full quality assessment review of articles was done before selection for final review. Two authors independently assessed the articles for inclusion in the review. Any discrepancy which arose between the authors in the review process was resolved through discussion for consensus.

\section{Eligibility criteria}

Studies were included in the review if:

- the design was cross-sectional, case-control, prospective or retrospective cohorts,
- it evaluated the link between at least one cardiovascular risk factor (chronic hypertension, diabetes, dyslipidemia, obesity, overweight, tobacco, alcohol) and preeclampsia.

- it was published in English or French.

We did not use a restriction on the years of publication. Only studies meeting our inclusion criteria were selected. The quality of retrieved studies was assessed using the Newcastle-Ottawa scale (NOS) [13]. Selection, comparability, and outcome/exposure assessment were rated for case-control and cohort studies separately. The rating system scores from 0 (highest degree of bias) to 9 (lowest degree of bias) allocated among three criteria: selection (0 to 4), comparability (0 to 2 ) and outcome/exposure assessment (0 to 3 ). Studies with a total score of 0 for one of the three criteria were excluded.

\section{Data extraction}

Selected studies were analyzed for data extraction. We extracted data concerning: authors, years and areas of studies, study populations, study design, sample size, sampling methods, inclusion and exclusion criteria, preeclampsia definition, risk factors, length of follow up for cohort studies and exposure variables for all studies. We also reported all studied variables per each article. Targeted risk factors were chronic hypertension, diabetes mellitus, overweight, obesity, alcohol, tobacco, and dyslipidemia. Preeclampsia was a main outcome reported in the included studies. Bias, confounding and their management were assessed in included studies by reviewing each study methods and discussion sections. Studies with significant bias were not included in this review. When available, results were reported as odds ratios or relative risks with $95 \%$ confidence intervals.

\section{Outcomes}

The main outcome was preeclampsia. It was defined as new onset of systolic blood pressure of $140 \mathrm{mmHg}$ or more and diastolic blood pressure of $90 \mathrm{mmHg}$ or more, at least twice on two occasions at least $4 \mathrm{~h}$ apart, with either $24 \mathrm{~h}$ proteinuria $\geq 300 \mathrm{mg}$ or urinary protein strip showing $\geq 1+$ without urinary tract infection, according to the American College of Obstetricians and Gynecologists, in almost all the included studies. However, one study used a higher blood pressure cut-off of 160/110 associated with random proteinuria $\geq 2+[14]$.

\section{Results}

A total of 770 articles were retrieved through electronic searching in databases and 4 studies from references. Of this, 44 duplicates were removed and 554 studies were removed because they were conducted in a region other than SSA, or preeclampsia (hypertensive disorders) was 
not a main outcome. One hundred and seventy-six titles and abstracts were screened. Finally, twelve studies (8 case-control, 3 cohorts and one cross-sectional) were included in this systematic review. The study selection process is summarized in Fig. 1.

Most studies had high quality case definition and subject selection. Cases and controls were selected from the same population. There was no information on the proportion of non-response and the representativeness of the sample in some studies. All included studies scored 7 and above at quality assessment. Table 1 and Table 2 present NOS-based evaluation of case-control and cohort studies, respectively.

\section{Studies description}

The characteristics of included studies are shown in Table 3. They were conducted between 1998 and 2018 . Participants were recruited during pregnancy or after delivery. Preeclampsia standards were not identical among all included studies. They were based either on the American College of Obstetrician and Gynecologists or the International Society for the Study of Hypertension in Pregnancy (ISSHP) standards. Only one study was conducted in a French-speaking SSA country. In cohort studies, pregnant women were included before 20 weeks of pregnancy. Case-control study populations mostly included women who were more than 20 weeks pregnant or those who had already given birth. Some cardiovascular risk factors such as dyslipidemia and tobacco use were not studied. Few studies assessed tobacco (4/12). Sample size was calculated in almost all the included studies. The odds ratios are summarized according to the risk factors in Table 4.

\section{Chronic hypertension}

Chronic hypertension increased the risk of developing preeclampsia by up to 10 -fold $[6,14,16,21]$. Some

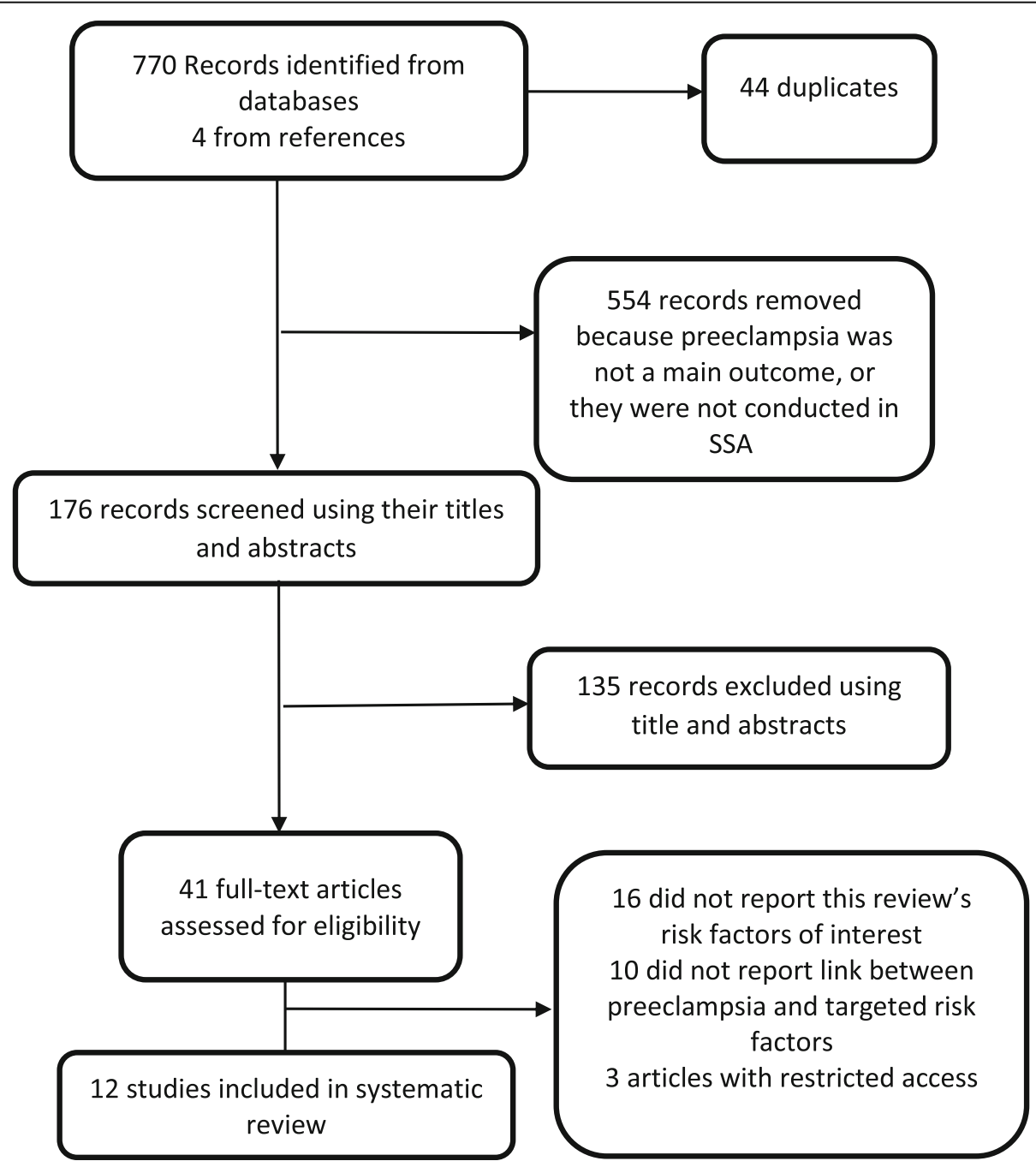

Fig. 1 Flow diagram for selection of studies included in systematic review 
Table 1 Quality assessment of included case-control studies

\begin{tabular}{|c|c|c|c|c|c|c|c|c|}
\hline & $\begin{array}{l}\text { Mahomed } \\
\text { et al. }\end{array}$ & $\begin{array}{l}\text { Anorlu } \\
\text { et al. }\end{array}$ & $\begin{array}{l}\text { Kiondo } \\
\text { et al. }\end{array}$ & $\begin{array}{l}\text { Guerrier } \\
\text { et al. }\end{array}$ & $\begin{array}{l}\text { Endeshaw } \\
\text { et al. }\end{array}$ & $\begin{array}{l}\text { Grum } \\
\text { et al. }\end{array}$ & $\begin{array}{l}\text { Kahsay } \\
\text { et al. }\end{array}$ & $\begin{array}{l}\text { Mrema } \\
\text { et al. }\end{array}$ \\
\hline \multicolumn{9}{|l|}{ Selection } \\
\hline Is the case definition adequate? & 1 & 1 & 1 & 1 & 1 & 1 & 1 & 1 \\
\hline Representativeness of cases & 1 & 1 & 1 & 1 & 1 & 1 & 0 & 1 \\
\hline Selection of Controls & 0 & 0 & 0 & 0 & 0 & 0 & 0 & 0 \\
\hline Definition of Controls & 1 & 1 & 1 & 1 & 1 & 1 & 1 & 1 \\
\hline \multicolumn{9}{|l|}{ Comparability } \\
\hline $\begin{array}{l}\text { Comparability of cases and controls based on } \\
\text { the design or analysis }\end{array}$ & 2 & 2 & 1 & 2 & 2 & 2 & 2 & 2 \\
\hline \multicolumn{9}{|l|}{ Exposure } \\
\hline Ascertainment of exposure & 1 & 1 & 1 & 1 & 1 & 1 & 1 & 1 \\
\hline $\begin{array}{l}\text { Same method of ascertainment for cases and } \\
\text { controls }\end{array}$ & 1 & 1 & 1 & 1 & 1 & 1 & 1 & 1 \\
\hline Non-Response rate & 0 & 1 & 1 & 0 & 1 & 1 & 1 & 0 \\
\hline Total & 7 & 8 & 7 & 7 & 8 & 8 & 7 & 7 \\
\hline
\end{tabular}

included studies did not evaluate for chronic hypertension $[15,18,19]$.

\section{Diabetes}

In our review, only one case-control study reported the role of pregestational diabetes in the occurrence of preeclampsia. Pregnant women with pre-gestational diabetes were four times at risk of developing preeclampsia [19]. Pregnant women with known diabetes before pregnancy were not included in some studies [22].

\section{Overweight and obesity}

Three case-control studies reported that being overweight before pregnancy increased the risk of developing preeclampsia by up to seven times $[10,15,22]$. All cohort studies [20-22] and two case-control studies [10,
17] in this review reported that obesity was an important risk factor for preeclampsia.

\section{Alcohol}

One case-control study suggested an association between alcohol and preeclampsia. In this study, women who reported alcohol consumption during pregnancy were more likely to develop preeclampsia compared to those who did not drink alcohol in multivariable analysis ( $\mathrm{aOR}=3.97,95 \% \mathrm{CI}=1.80-8.75)$ [18]. However, Kiondo et al. found no association between alcohol and preeclampsia [16].

\section{Discussion}

The prevalence of cardiovascular risk factors in SSA women is not negligeable. The median prevalences were:

Table 2 Quality assessment of included cohort studies

\begin{tabular}{|c|c|c|c|}
\hline & Singh et al. & Baragou et al. & Musa et al. \\
\hline \multicolumn{4}{|l|}{ Selection } \\
\hline Representativeness of the exposed cohort & 1 & 1 & 1 \\
\hline Selection of the non-exposed cohort & 0 & 1 & 1 \\
\hline Ascertainment of exposure & 1 & 1 & 1 \\
\hline Demonstration that outcome of interest was not present at start of study & 1 & 1 & 1 \\
\hline \multicolumn{4}{|l|}{ Comparability } \\
\hline Comparability of cohorts based on the design or analysis & 2 & 2 & 2 \\
\hline \multicolumn{4}{|l|}{ Outcome } \\
\hline Assessment of outcome & 1 & 1 & 1 \\
\hline Was follow-up long enough for outcomes to occur? & 1 & 1 & 1 \\
\hline Adequacy of follow-up of cohorts & 1 & 1 & 1 \\
\hline Total & 8 & 9 & 9 \\
\hline
\end{tabular}


Table 3 Characteristics and risk factors reported in included studies

\begin{tabular}{|c|c|c|c|c|c|c|c|}
\hline References & Setting & $\mathbf{N}$ & Cases & Study Population & PE assessment & Predictor variables & Risk factors \\
\hline \multicolumn{8}{|l|}{ Case controls } \\
\hline $\begin{array}{l}\text { Mohamed } \\
\text { et al.1998 } \\
\text { [15] }\end{array}$ & $\begin{array}{l}\text { Harare, } \\
\text { Zimbabwe }\end{array}$ & 338 & $\begin{array}{l}144 \text { cases, } \\
194 \\
\text { controls }\end{array}$ & $\begin{array}{l}\text { Women who delivered at } \\
\text { Harare hospital maternity } \\
\text { from June } 1995 \text { to April } \\
\text { 1996, recruited from } \\
\text { admission and birth } \\
\text { records. } \\
\text { Controls were } \\
\text { normotensive pregnant } \\
\text { women delivering within } \\
\text { two hours and were } \\
\text { matched in childbirth } \\
\text { mode with the case. }\end{array}$ & $\begin{array}{l}\text { American College of } \\
\text { Obstetricians and } \\
\text { Gynecologists definition } \\
\text { Blood pressure }>140 / 90 \\
\text { mmHg at least twice and } \\
\text { occasional proteinuria }>1 \\
\text { g/l at least twice at } 4 \mathrm{~h} \\
\text { intervals. }\end{array}$ & Age, overweight, obesity. & $\begin{array}{l}\text { Overweight } \\
\text { OR } 7(2.9- \\
19.4)\end{array}$ \\
\hline $\begin{array}{l}\text { Anorlu } \\
\text { et al. } 2005 \\
{[6]}\end{array}$ & $\begin{array}{l}\text { Lagos, } \\
\text { Nigeria }\end{array}$ & 368 & $\begin{array}{l}128 \text { cases, } \\
240 \\
\text { controls }\end{array}$ & $\begin{array}{l}\text { Delivered women at Lagos } \\
\text { university hospital from } \\
\text { February } 2001 \text { to August } \\
2002 \text { with preeclampsia or } \\
\text { eclampsia. } \\
\text { The randomly selected } \\
\text { controls were women who } \\
\text { gave birth at } 24-48 \mathrm{~h} \\
\text { intervals without } \\
\text { preeclampsia. For each } \\
\text { case, two controls were } \\
\text { selected. }\end{array}$ & $\begin{array}{l}\text { Blood pressure }>140 / 90 \\
\text { mmHg at least twice on } \\
\text { two occasions at least } 4 \mathrm{~h} \\
\text { apart with either } 24 \mathrm{~h} \\
\text { proteinuria } \geq 300 \mathrm{mg} \text { or } \\
\text { urinary protein strip with } \\
\geq 1+\text { protein in the } \\
\text { absence of urinary tract } \\
\text { infections. }\end{array}$ & $\begin{array}{l}\text { Age, parity, educational } \\
\text { level, occupation, home } \\
\text { and work environment, } \\
\text { family history of } \\
\text { hypertension, chronic } \\
\text { hypertension, previous } \\
\text { preeclampsia. }\end{array}$ & $\begin{array}{l}\text { Chronic } \\
\text { hypertension } \\
\text { OR } \mathbf{2 . 2 1} \\
(1.17-6.20)\end{array}$ \\
\hline $\begin{array}{l}\text { Kiondo } \\
\text { et al. } 2012 \\
{[16]}\end{array}$ & $\begin{array}{l}\text { Mulago, } \\
\text { Uganda }\end{array}$ & 557 & $\begin{array}{l}207 \text { cases, } \\
352 \\
\text { controls }\end{array}$ & $\begin{array}{l}\text { Pregnant women aged } 15 \\
\text { to } 39 \text { randomly selected } \\
\text { from computer database } \\
\text { from May 1, } 2008 \text { to May } \\
\text { 1, } 2009 \text { living within } 15 \mathrm{~km} \\
\text { of Mulago hospital and } \\
\text { more than } 20 \text { weeks } \\
\text { pregnant. } \\
\text { Women with cardiac } \\
\text { disease, sickle cell disease, } \\
\text { eclampsia, HELLP } \\
\text { syndrome and renal failure } \\
\text { were excluded from } \\
\text { controls. For each } \\
\text { identified case, two } \\
\text { controls were selected on } \\
\text { the same day until the } \\
\text { required number of } \\
\text { patients was obtained. }\end{array}$ & $\begin{array}{l}\text { Blood pressure }>140 / 90 \\
\mathrm{mmHg} \text { at least twice on } \\
\text { two occasions at least } 4 \mathrm{~h} \\
\text { apart with either } 24 \mathrm{~h} \\
\text { proteinuria } \geq 300 \mathrm{mg} \text { or } \\
\text { random proteinuria } \geq 1+\end{array}$ & $\begin{array}{l}\text { Age, educational level, } \\
\text { marital status, smoking } \\
\text { status, alcohol intake, } \\
\text { MUAC, history of diabetes, } \\
\text { hypertension, family } \\
\text { history of hypertension, } \\
\text { parity. }\end{array}$ & $\begin{array}{l}\text { Chronic } \\
\text { hypertension } \\
\text { OR } \mathbf{2 . 2 9} \mathrm{Cl} \\
(1.12-4.66)\end{array}$ \\
\hline $\begin{array}{l}\text { Guerrier } \\
\text { et al. } 2013 \\
\text { [14] }\end{array}$ & $\begin{array}{l}\text { Jahun, } \\
\text { Nigeria }\end{array}$ & 1676 & $\begin{array}{l}419 \text { cases } \\
\text { (175 severe } \\
\text { PE, } 244 \\
\text { eclampsia), } \\
1257 \\
\text { controls }\end{array}$ & $\begin{array}{l}\text { Pregnant women admitted } \\
\text { from October } 2010 \text { to May } \\
2011 .\end{array}$ & $\begin{array}{l}\text { Severe PE: Blood } \\
\text { pressure }>160 / 110 \mathrm{mmHg} \\
\text { and random proteinuria } \\
\geq 2+\end{array}$ & $\begin{array}{l}\text { Age, ethnicity, occupation, } \\
\text { personal history of PE, } \\
\text { history of hypertension, } \\
\text { family history of PE and } \\
\text { hypertension, number of } \\
\text { ANC visits. }\end{array}$ & $\begin{array}{l}\text { Chronic } \\
\text { hypertension } \\
\text { OR } \mathbf{1 0 . 5}(5.8- \\
\text { 19) }\end{array}$ \\
\hline $\begin{array}{l}\text { Endeshaw } \\
\text { et al. } 2016 \\
{[17]}\end{array}$ & $\begin{array}{l}\text { Bahir Dar, } \\
\text { Ethiopia }\end{array}$ & 453 & $\begin{array}{l}151 \text { cases, } \\
302 \\
\text { controls }\end{array}$ & $\begin{array}{l}\text { Pregnant women } \\
\text { attending or delivered in a } \\
\text { referral hospital or six } \\
\text { health centers in Bahir Dar } \\
\text { between June and } \\
\text { September 1994, } \\
\text { diagnosed pre-eclamptic } \\
\text { by a physician or midwife. } \\
\text { For each case, two } \\
\text { controls were randomly } \\
\text { selected in the same } \\
\text { center on the same day. }\end{array}$ & $\begin{array}{l}\text { Blood pressure > 140/90 } \\
\text { mmHg at least twice on } \\
\text { two occasions and } \\
\text { laboratory-confirmed } \\
\text { proteinuria }\end{array}$ & $\begin{array}{l}\text { Age, area of residence, } \\
\text { marital status, occupation, } \\
\text { MUAC, fruit, vegetable, } \\
\text { folate, alcohol and coffee } \\
\text { intake, anemia, physical } \\
\text { activity. }\end{array}$ & $\begin{array}{l}\text { Obesity } \\
\text { OR } \mathbf{3 . 3 3} \\
(1.87-5.79)\end{array}$ \\
\hline $\begin{array}{l}\text { Grum } \\
\text { et al. } 2017 \\
\text { [18] }\end{array}$ & $\begin{array}{l}\text { Addis } \\
\text { Ababa, } \\
\text { Ethiopia }\end{array}$ & 291 & $\begin{array}{l}97 \text { cases, } \\
194 \\
\text { controls }\end{array}$ & $\begin{array}{l}\text { Pregnant women } \\
\text { attending the two largest } \\
\text { hospitals of Addis Ababa }\end{array}$ & $\begin{array}{l}\text { Blood pressure }>140 / 90 \\
\mathrm{mmHg} \text { at least twice on } \\
\text { two occasions with either }\end{array}$ & $\begin{array}{l}\text { Age, ethnicity, religion, } \\
\text { marital status, occupation, } \\
\text { educational status, family }\end{array}$ & $\begin{array}{l}\text { Alcohol } \\
\text { consumption } \\
\text { OR } \mathbf{3 . 9 7}\left(1.8^{-}\right.\end{array}$ \\
\hline
\end{tabular}


Table 3 Characteristics and risk factors reported in included studies (Continued)

\begin{tabular}{|c|c|c|c|c|c|c|c|}
\hline References & Setting & $\mathbf{N}$ & Cases & Study Population & PE assessment & Predictor variables & Risk factors \\
\hline & & & & $\begin{array}{l}\text { from December } 2015 \text { to } \\
\text { February } 2016 \text { diagnosed } \\
\text { pre-eclamptic by an ob- } \\
\text { stetrician. Women with } \\
\text { known hypertension, renal } \\
\text { disease, with serious med- } \\
\text { ical conditions and those } \\
\text { who could not give con- } \\
\text { sent were excluded. } \\
\text { Controls were women not } \\
\text { diagnosed with } \\
\text { preeclampsia in the two } \\
\text { hospitals. }\end{array}$ & $\begin{array}{l}24 \mathrm{~h} \text { proteinuria } \geq 300 \mathrm{mg} \\
\text { or random proteinuria } \\
\geq 1+\text { after } 28 \text { weeks } \\
\text { gestation }\end{array}$ & $\begin{array}{l}\text { history of hypertension, } \\
\text { history of eclampsia, } \\
\text { gravidity, multiple } \\
\text { pregnancy, alcohol, fruit } \\
\text { intake. }\end{array}$ & $8.75)$ \\
\hline $\begin{array}{l}\text { Kahsay } \\
\text { et al. } 2018 \\
\text { [19] }\end{array}$ & $\begin{array}{l}\text { Tigray, } \\
\text { Ethiopia }\end{array}$ & 330 & $\begin{array}{l}110 \text { cases, } \\
220 \\
\text { controls }\end{array}$ & $\begin{array}{l}\text { Pregnant women with } \\
\text { gestational hypertension } \\
\text { or PE diagnosed by an } \\
\text { obstetrician in one of the } \\
\text { seven hospitals in the } \\
\text { Tigray region from June to } \\
\text { November } 2017 \text {. } \\
\text { Women without } \\
\text { hypertension were } \\
\text { controls, women less than } \\
20 \text { weeks pregnant were } \\
\text { excluded. Cases and } \\
\text { controls were matched for } \\
\text { parity and time of arrival at } \\
\text { hospital. }\end{array}$ & $\begin{array}{l}\text { Blood pressure } \geq 140 / 90 \\
\mathrm{mmHg} \text { at least twice } \\
\text { associated with } \\
\text { proteinuria } \geq 1+\text { on the } \\
\text { urine test strip }\end{array}$ & $\begin{array}{l}\text { Age, area of residence, } \\
\text { marital status, occupation, } \\
\text { ethnicity, income category, } \\
\text { family history of } \\
\text { hypertension, MUAC, BMI, } \\
\text { fruit and vegetable intake, } \\
\text { coffee use, history of } \\
\text { smoking, diabetes mellitus, } \\
\text { oral contraceptive use. }\end{array}$ & $\begin{array}{l}\text { Overweight } \\
\text { OR } \mathbf{5 . 5}(1.12- \\
27.6 \\
\text { Diabetes OR } \\
\mathbf{5 . 4}(1.1-27.0)\end{array}$ \\
\hline $\begin{array}{l}\text { Mrema } \\
\text { et al. } 2018 \\
{[10]}\end{array}$ & $\begin{array}{l}\text { Kilimanjaro, } \\
\text { Tanzania }\end{array}$ & $\begin{array}{l}17 \\
738\end{array}$ & $\begin{array}{l}582 \text { cases, } \\
17,156 \\
\text { controls }\end{array}$ & $\begin{array}{l}\text { Birth records selected } \\
\text { women with one previous } \\
\text { pregnancy and a } \\
\text { monofetal actual } \\
\text { pregnancy diagnosed as } \\
\text { pre-eclamptic by an } \\
\text { obstetrician. } \\
\text { Normotensives pregnant } \\
\text { women were controls. }\end{array}$ & $\begin{array}{l}\text { Blood pressure }>140 / 90 \\
\mathrm{mmHg} \\
\text { associated with } 24 \mathrm{~h} \\
\text { proteinuria } \geq 300 \mathrm{mg}\end{array}$ & $\begin{array}{l}\text { Age, marital status, } \\
\text { occupation, educational } \\
\text { level, pregnancy number, } \\
\text { number of ANC, chronic } \\
\text { hypertension, diabetes, } \\
\text { heart disease, BMI. }\end{array}$ & $\begin{array}{l}\text { Overweight } \\
\text { OR } \mathbf{1 . 4}(1.2- \\
1.8) \\
\text { Obesity OR } \\
\mathbf{1 . 8}(1.3-2.4)\end{array}$ \\
\hline \multicolumn{8}{|c|}{ Cohort studies } \\
\hline $\begin{array}{l}\text { Singh } \\
\text { et al. } 2014 \\
{[20]}\end{array}$ & $\begin{array}{l}\text { Sokoto, } \\
\text { Nigeria }\end{array}$ & 216 & 13 PE (6\%) & $\begin{array}{l}\text { Pregnant women attended } \\
\text { Usmanu Danfodiyo } \\
\text { University hospital from } \\
\text { March to December } 2011 \\
\text { at less than } 20 \text { weeks } \\
\text { pregnancy and were } \\
\text { followed-up to } 6 \text { weeks } \\
\text { after giving birth. The main } \\
\text { outcome was the develop- } \\
\text { ment of hypertensive dis- } \\
\text { orders during the follow- } \\
\text { up period. }\end{array}$ & $\begin{array}{l}\text { Blood pressure > 140/80 } \\
\mathrm{mmHg} \text { associated with } \\
\text { proteinuria }\end{array}$ & $\begin{array}{l}\text { Age, marital status, } \\
\text { education, occupation, } \\
\text { type of gestation, family } \\
\text { history of hypertension } \\
\text { and diabetes mellitus, } \\
\text { history of preeclampsia, } \\
\text { BMI. }\end{array}$ & $\begin{array}{l}\text { Obesity RR } \\
\mathbf{2 . 7}(1.3-5.7)\end{array}$ \\
\hline $\begin{array}{l}\text { Baragou } \\
\text { et al. } 2014 \\
{[21]}\end{array}$ & $\begin{array}{l}\text { Lomé, Togo } \\
\text { Urban }\end{array}$ & 1620 & $\begin{array}{l}114 \mathrm{PE} \\
(7 \%)\end{array}$ & $\begin{array}{l}\text { Pregnant women recruited } \\
\text { in the gynecology service } \\
\text { of Tokoin University } \\
\text { hospital from October 1, } \\
2011 \text { to September 31, } \\
2012 \text {. The main outcome } \\
\text { was hypertensive disorder } \\
\text { during pregnancy. Women } \\
\text { were followed during the } \\
\text { pregnancy to } 3 \text { months } \\
\text { postpartum. }\end{array}$ & $\begin{array}{l}\text { Blood pressure }>140 / 90 \\
\text { mmHg at least twice on } \\
\text { two occasions at least } 4 \mathrm{~h} \\
\text { apart with either } 24 \mathrm{~h} \\
\text { proteinuria } \geq 300 \mathrm{mg} \text { or } \\
\text { urinary protein strip with } \\
\geq 1+\text { protein in the } \\
\text { absence of urinary tract } \\
\text { infection }\end{array}$ & $\begin{array}{l}\text { Age, occupation, multiple } \\
\text { pregnancy, stress, obesity, } \\
\text { familial history of } \\
\text { hypertension, history of } \\
\text { preeclampsia. }\end{array}$ & $\begin{array}{l}\text { Chronic } \\
\text { hypertension } \\
\text { RR } \mathbf{3} \\
\text { Obesity RR } \\
\mathbf{2 . 8}\end{array}$ \\
\hline $\begin{array}{l}\text { Musa et al. } \\
2018 \text { [22] }\end{array}$ & Jos, Nigeria & 307 & $\begin{array}{l}27 \mathrm{PE} \\
(8.8 \%)\end{array}$ & $\begin{array}{l}\text { Pregnant women } \\
\text { attending the Jos } \\
\text { University hospital } \\
\text { between November } 2010\end{array}$ & $\begin{array}{l}\text { Blood pressure } \geq 140 / 90 \\
\mathrm{mmHg} \text { at least twice } \\
\text { associated with } \\
\text { proteinuria } \geq 2+\text { on the }\end{array}$ & $\begin{array}{l}\text { Age, gestational age, BMI, } \\
\text { previous PE, miscarriages, } \\
\text { parity, history of infertility, } \\
\text { HIV status. }\end{array}$ & $\begin{array}{l}\text { Obesity RR } \\
\mathbf{3 . 9}(1.5-10.0)\end{array}$ \\
\hline
\end{tabular}


Table 3 Characteristics and risk factors reported in included studies (Continued)

\begin{tabular}{|c|c|c|c|c|c|c|c|}
\hline References & Setting & $\mathbf{N}$ & Cases & Study Population & PE assessment & Predictor variables & Risk factors \\
\hline & & & & $\begin{array}{l}\text { to August } 2011 \text { before } 20 \\
\text { weeks pregnancy without } \\
\text { any signs of PE. Patients } \\
\text { with chronic hypertension, } \\
\text { proteinuria, chronic renal } \\
\text { failure, diabetes, sickle cell } \\
\text { disease were not included. } \\
\text { The primary outcome was } \\
\text { preeclampsia and follow- } \\
\text { up was terminated at any } \\
\text { gestational age if the } \\
\text { woman developed pre- } \\
\text { eclampsia or delivery of } \\
\text { her baby with or without } \\
\text { development of } \\
\text { preeclampsia. }\end{array}$ & urine test strip & & \\
\hline $\begin{array}{l}\text { Tessama } \\
\text { et al. } 2013 \\
{[23]}\end{array}$ & $\begin{array}{l}\text { Dessie, } \\
\text { Ethiopia }\end{array}$ & 475 & $\begin{array}{l}41 \mathrm{PE} \\
(8.6 \%)\end{array}$ & $\begin{array}{l}\text { Pregnant women } \\
\text { attending prenatal visits in } \\
\text { Dessie Hospital between } \\
\text { August and September } \\
2013 \text { at more than } 20 \\
\text { weeks of pregnancy. }\end{array}$ & $\begin{array}{l}\text { Blood pressure }>140 / 90 \\
\text { mmHg associated with } \\
\text { proteinuria } \geq 1+\text { on the } \\
\text { urine test strip }\end{array}$ & $\begin{array}{l}\text { Age, marital status, } \\
\text { ethnicity, educational level, } \\
\text { tobacco, alcohol use, } \\
\text { family history of } \\
\text { hypertension and diabetes } \\
\text { mellitus, history of } \\
\text { hypertension, gravidity, } \\
\text { parity. }\end{array}$ & $\begin{array}{l}\text { Chronic } \\
\text { hypertension } \\
\text { OR } \mathbf{4 . 3}(1,33- \\
13,9)\end{array}$ \\
\hline
\end{tabular}

PE Preeclampsia, OR Odds Ratio, RR Risk Ratio

hypertension (29\%), diabetes (7\%), overweight (35\%), obesity (11\%), alcohol consumption (13\%), tobacco (2\%) [24-26]. Apart from tobacco, there is a significant prevalence of cardiovascular risk factors in SSA women.

This review presents evidence from published observational studies on the association between cardiovascular risk factors and preeclampsia. Chronic hypertension can increase the risk of developing preeclampsia during pregnancy by 3 to 10 -fold. This association was also found among Latin American and Caribbean women and is generally present in LMIC $[8,27]$. A possible explanation for this is the lack of follow-up of chronic hypertension among women. These results emphasize the need for detecting and managing hypertension among women in SSA.

Other high-risk factors for preeclampsia are overweight and obesity. These findings corroborate Proorolajal's et al. meta-analysis, which concluded that overweight and obesity are predictors of preeclampsia. Paré et al., in a prospective cohort study, reported that

Table 4 Reported Odds Ratios according to each risk factor

\begin{tabular}{llll}
\hline Risk factors & Number of studies & Odds Ratios/Relative Risk & 95\% Confidence Intervals \\
\hline Chronic hypertension & 5 & $\mathbf{2 . 2}$ & $1.17-6.20$ \\
& & $\mathbf{2 . 3}$ & $1.12-4.66$ \\
& & $\mathbf{1 0 . 5}$ & $5.8-19$ \\
& & $\mathbf{4 . 3}$ & $1.33-13.9$ \\
& 5 & $\mathbf{3 . 0}$ & $1.87-5.79$ \\
Obesity & $\mathbf{3 . 3}$ & $1.3-2.4$ \\
& & $\mathbf{1 . 8}$ & $1.3-5.7$ \\
& & $\mathbf{2 . 7}$ & $1.5-10.0$ \\
Overweight & $\mathbf{2 . 8}$ & $2.9-19.4$ \\
& 3 & $\mathbf{3 . 9}$ & $1.12-27.6$ \\
Diabetes & $\mathbf{7 . 0}$ & $\mathbf{5 . 5}$ & $1.2-1.8$ \\
Alcohol & 1 & $\mathbf{1 . 4}$ & $1.1-27.0$ \\
\hline
\end{tabular}


the risk of preeclampsia increases with the body mass index $[7,28]$. These results require clarification, as the BMI in the pregestational period could not be evaluated in most cases. In LMIC, women are not always able to accurately report their body mass index or weight, so some authors have used mid-upper arm circumference to assess nutritional status, because this parameter may be stable during pregnancy [29]. This methodological particularity could lead to an overestimation of obesity cases, because pregnancy is responsible for weight gain in most women. Other authors have evaluated BMI based on the weight reported by pregnant women, which is an important methodological limitation. Obesity is associated with oxidative stress, increased levels of circulating inflammation markers, dyslipidemia, insulin resistance and impaired endothelial function. These metabolic and biochemical disturbances likely predispose to an intrauterine environment favorable to placental perfusion disorders and endothelial dysfunction in preeclampsia [30].

Although only one study reported a significant association between pre-gestational diabetes and preeclampsia, our findings are similar to those reported by Duckitt et al. in a systematic review. Being diabetic before pregnancy increases the risk of preeclampsia by 4 times [31]. However, one study alone does not permit generalizing these findings. Like diabetes, alcohol consumption and dyslipidemia have been poorly studied. Both factors may increase the risk of preeclampsia [18]. However, Kiondo et al. found no association between alcohol consumption and preeclampsia in Uganda [16]. These results may be explained by the fact that alcohol consumption was selfreported, and in SSA and worldwide, alcohol consumption by pregnant women is not considered acceptable, or is even prohibited. No studies evaluated the impact of tobacco on the occurrence of preeclampsia. This may be explained by the fact that in SSA, tobacco use is not common among women. However, tobacco in its smoking form reduces the risk of preeclampsia by $35 \%$, although the mechanism remains controversial [32]. This protective effect of smoking on preeclampsia risk is limited to low-risk pregnancies only. Smoking is an independent risk factor for superimposed preeclampsia in chronic hypertension, which is significant in the context of other cardiovascular risk factors [33]. Despite this lack of information, the harmful effects of tobacco on health, especially in arteriosclerosis, should not be neglected.

In LMIC, there is an epidemiological transition from communicable disease to non-communicable disease, led by cardiovascular disease, which accounted for 33.2\% of deaths worldwide in 2008 [26]. Since cardiovascular risk factors are involved in the occurrence of preeclampsia, the screening of women at risk could help reduce mortality related to hypertensive complications of pregnancy, in particular preeclampsia. Reducing preeclampsia and cardiovascular risk factors would lessen long-term cardiovascular complications and therefore female mortality linked to non-communicable disease. Indeed, the occurrence of preeclampsia can increase by four the risk of heart failure and by two the risk of stroke, coronary heart disease or death from a cardiovascular event in the medium and long term.

However, this review has some limitations. The studies were all conducted in hospital settings. Therefore, there might be a selection bias because of home deliveries. The lack of studies in French speaking Sub-Saharan African countries does not permit generalizing our results. The self-reported data collected in the studies does not guarantee the reliability of the results. Some authors did not consider possible confounding factors that could lead to misestimation of their results. We included studies from various geographical areas, conducted with specific methodologies. One limitation of our review is that we report results from studies with potential limitations and which did not focus exclusively on preeclampsia.

\section{Conclusion}

Cardiovascular risk factors are involved in the occurrence of preeclampsia in SSA. This review highlights the role of chronic hypertension, diabetes, overweight, obesity, alcohol. Some of the included studies have methodological limitations. The lack of data suggests a need for prospective cohort studies to assess the impact of cardiovascular risk factors on the occurrence of preeclampsia in SSA. Pre-pregnancy cardiovascular risk factor screening is needed to prevent preeclampsia among women.

\section{Abbreviations}

SSA: Sub-Saharan Africa; LMIC: Low- and middle-income countries; NOS: Newcastle-Ottawa scale; WHO: World health Organization; PRIS MA: Preferred reporting items for systematic reviews and meta-analyses; ISSHP: International society for the study of hypertension in pregnancy; BMl: Body mass index

\section{Acknowledgements}

Not applicable.

\section{Authors' contributions}

$\mathrm{OIH}$ : Data collection, Data analysis, Manuscript writing. SAA: Project development, Data analysis, Manuscript writing. YCH: Project development, Manuscript writing. PL: Project development, Manuscript writing. PMP: Manuscript writing. DSH: Project development, Data analysis. HB: Project development, Data analysis, Manuscript writing. All authors have read and approved the manuscript.

\section{Funding}

None.

\section{Availability of data and materials}

The datasets used and analyzed during the current study are available from the corresponding author on reasonable request.

Ethics approval and consent to participate

Non applicable. 


\section{Consent for publication}

Non applicable.

\section{Competing interests}

The authors declare that they have no competing interests.

\section{Author details}

'INSERM, U1094, Tropical Neuroepidemiology, Limoges, France. ${ }^{2}$ Univ. Limoges, U1094, Tropical Neuroepidemiology, Institute of Epidemiology and Tropical Neurology, GEIST, Limoges, France. ${ }^{3}$ IRD, Associated Unit, Tropical Neuroepidemiology, Limoges, France. ${ }^{4}$ School of Health Sciences, Laboratory of Chronic Diseases Epidemiology (LEMACEN), University Abomey-Calavi, Cotonou, Benin. ${ }^{5}$ Department of Vascular Medicine-Vascular Surgery, CHU Limoges, Limoges, France. ${ }^{6}$ Department of Internal Medicine, CHU Limoges, Limoges, France.

Received: 29 April 2020 Accepted: 18 January 2021

Published online: 30 January 2021

\section{References}

1. World Health Organization. Strategies towards ending preventable maternal mortality (EPMM). Geneva: World Health Organization; 2015 p. 44. Available from: https://apps.who.int/iris/handle/10665/153544. ISBN: 9789241508483.

2. World Health Organization. Trends in maternal mortality: 1990 to 2015 : estimates by WHO, UNICEF, UNFPA, World Bank Group and the United Nations population division. Geneva: WHO press; 2015.

3. Say L, Chou D, Gemmill A, Moller AB, Daniels J, Temmerman M, et al. Global causes of maternal death: a WHO systematic analysis. Lancet Glob Health. 2014;2(6):e323-33.

4. Mol BWJ, Roberts CT, Thangaratinam S, Magee LA, de Groot CJM, Hofmeyr GJ. Pre-eclampsia. Lancet. 2016;387:999-1011.

5. Shamsi U, Saleem S, Nishter N. Epidemiology and risk factors of preeclampsia; an overview of observational studies. Al Ameen J Med Sci. 2013;6(4):292-300.

6. Anorlu RI, Iwuala NC, Odum Cl. Risk factors for pre-eclampsia in Lagos, Nigeria. Aust N Z J Obstet Gynaecol. 2005;45:278-82.

7. Paré E, Parry S, McElrath FT, Pucy D, Newton A, Lim K-H. Clinical risk factors for preeclampsia in 21st century. Obstet Gynecol. 2014;14(4):763-70.

8. Bilano VL, Ota E, Ganchimeg T, Mori R, Paulo SJ. Risk factors of preEclampsia/Eclampsia and its adverse outcomes in low- and middle-income countries: a WHO secondary analysis. PLoS One. 2014;9(3):e91198.

9. Merviel P, Touzart L, Deslandesa V, Delmasb M, Coicaud M, Gondry J. Facteurs de risque de la prééclampsie en cas de grossesse unique. J Gynécol Obstét Biol Reprod (Paris). 2008;37:477-82.

10. Mrema D, Lie RT, Østbye T, Mahande MJ, Kjersti Daltveit A. The association between pre pregnancy body mass index and risk of preeclampsia: a registry based study from Tanzania. BMC Pregnancy Childbirth 2018; 18:56. https://doi.org/https://doi.org/10.1186/s12884-018-1687-3.

11. Baumfeld $Y$, Novack L, Wiznitzer A, Sheiner E, Henkin $Y$, Sherf M et al. Preconception dyslipidemia is associated with development of preeclampsia and gestational diabetes mellitus. PLoS One 2015; 10(11): e0139164. https:// doi.org/https://doi.org/10.1371/journal.pone.0142462.

12. Gedda M. French translation of the PRISMA reporting guidelines for writing and reading systematic reviews and meta-analyses. Kinésithér Rev 2015;15: 39-44. https://doi.org/https://doi.org/10.1016/j.kine.2014.11.004.

13. Wells GA, Shea B, O'Connell D, Peterson J, Welch V, Losos M, Tugwell P. The Newcastle-Ottawa Scale (NOS) for assessing the quality of nonrandomised studies in meta-analyses [Internet]. 2019. [Cited February 12, 2020]. Available from: http://www.ohri.ca/programs/clinical_epidemiology/oxford.asp

14. Guerrier G, Oluyide B, Keramarou M, Grais RF. Factors associated with severe preeclampsia and eclampsia in Jahun, Nigeria. Int J Womens Health. 2013;5: 509-13.

15. Mohamed K, William MA, Woelk GB, Jenkins-Woelk L, Mudzamiri S, Longstaff $L$, Sorensen TK. Risk factors for pre-eclampsia among Zimbabwean women: maternal arm circumference and other anthropometric measures of obesity. Paediatr Perinat Epidemiol. 1998;12:253-62.

16. Kiondo P, Wamuyu-Maina G, Bimenya GS, Tumwesigye NM, Wandabwa J, Okong P. Risk factors for pre-eclampsia in Mulago hospital, Kampala, Uganda. Trop Med Int Health. 2012;17(4):480-7.
17. Endeshaw M, Abebe F, Worku S, Menber L, Assress M, Assefa M. Obesity in young age is a risk factor for preeclampsia: a facility based case-control study, Northwest Ethiopia. BMC Pregnancy Childbirth. 2016;16:237.

18. Grum T, Seifu A, Abay M, Angesom T, Tsegay L. Determinants of preeclampsia/Eclampsia among women attending delivery Services in Selected Public Hospitals of Addis Ababa, Ethiopia: a case control study. Pregnancy Childbirth. 2017;17:307.

19. Kahsay BH, Gashe FE, Ayele WM. Risk factors for hypertensive disorders of pregnancy among mothers in Tigray region, Ethiopia: matched case control study. BMC Pregnancy and Childbirth. 2018;18:482

20. Singh S, Ahmed EB, Egondu SC, Ikechukwu NE. Hypertensive disorders in pregnancy among pregnant women in a Nigerian teaching hospital. Niger Med J. 2014:55(5):384-8.

21. Baragou S, Goeh-Akue E, Pio M, Afassinou YM, Atta B. Hypertension artérielle et grossesse à Lomé (Afrique sub-saharienne): aspects épidémiologiques, diagnostiques et facteurs de risque. Ann Cardiol Angeiol. 2014;63(3):145-50 https://doi.org/10.1016/j.ancard.2014.05.006

22. Musa J, Mohammed C, Ocheke A, Kahansim M, Pam V, Daru P. Incidence and risk factors for pre-eclampsia in Jos Nigeria. Afri Health Sci. 2018;18(3): 584-95.

23. Tessema GA, Tekeste A, Ayele TA. Preeclampsia and associated factors among pregnant women attending antenatal care in Dessie referral hospital, Northeast Ethiopia: a hospital-based study. BMC Pregnancy Childbirth. 2015:15:73.

24. Bretelle F, Sabatier F, Shojai R, Agostini A, Dignat-George F, Blanc B, et al. Avancées dans la physiopathologie de la pré-éclampsie : place de la réponse inflammatoire. Gynecol Obstet Fertil. 2004;32:482-9.

25. World Health Organization - African Region. Report on the status of major health risk factors for noncommunicable diseases: WHO African Region, 2015 [Internet]. Brazzaville: World Health Organization - Regional Office for Africa; 2015 p. 88. Available from: https://www.afro.who.int/publications/ report-status-major-health-risk-factors-noncommunicable-diseases-whoafrican-region-0. ISBN: 978-929023301-5.

26. NCD Alliance. Non communicable diseases: a priority for women's health and development. Geneva: NCD Alliance; 2011 p. 20. Available from: https:// ncdalliance.org/resources/noncommunicable-diseases-a-priority-forwomen\%E2\%80\%99s-health-and-development.

27. Conde-Agudelo A, Belizan J. M. Risk factors for pre-eclampsia in a large cohort of Latin American and Caribbean women. BJOG. 2000;107(1):75-83.

28. Poorolajal J, Jenabi E. The association between body mass index and preeclampsia: a meta-analysis. J Matern Fetal Neonatal Med. 2016;29(22):3670-6.

29. Ververs MT, Antierens A, Sackl A, Staderini N, Captier V. Which anthropometric indicators identify a pregnant woman as acutely malnourished and predict adverse birth outcomes in the humanitarian context? PLoS Curr. 2013;5:ecurrents.dis. 54a8b618c1bc031ea140e3f2934599c8. https://doi.org/10.1371/currents.dis. 54a8b618c1bc031ea140e3f2934599c8. PMID: 23787989; PMCID: PMC3682760.

30. Aliyu MH, Luke S, Kristensen S, Alio A, Salihu H. Joint effect of obesity and teenage pregnancy on the risk of preeclampsia: a population-based study. J Adolesc Health. 2010;46:77-82

31. Duckitt K, Harrington D. Risk factors for pre-eclampsia at antenatal booking: systematic review of controlled studies. BMJ 2005; 330: 565 http://dx.doi. org/https://doi.org/10.1136/bmj.38380.674340.EO

32. Wikstrom A, Stephansson O, Cnattingius S. Tobacco use during pregnancy and preeclampsia risk effects of cigarette smoking and snuff. Hypertension. 2010:55:1254-9.

33. Chappell LC, Enye S, Seed P, Briley AL, Poston L, Shennan AH. Adverse perinatal outcomes and risk factors for preeclampsia in women with chronic hypertension: a prospective study. Hypertension. 2008;51(4):1002-9. https://doi.org/10.1161/HYPERTENSIONAHA.107.107565.

\section{Publisher's Note}

Springer Nature remains neutral with regard to jurisdictional claims in published maps and institutional affiliations. 